\title{
Symmetry - Asymmetry of Generic Structure Order of the "Worldview" as Seen by the Russians, Italians and Frenchmen
}

\author{
Vadim A. Dorofeev \\ PhD in Psychology, Associate Professor, Psychology of Management and \\ Acmeology Department, Southern Federal University \\ Email: ya.fagot2011@yandex.ru \\ Julia A. Mochalova \\ PhD in Psychology, Associate Professor of faculty of Psychology of \\ Education, Southern Federal University \\ Email: guliya@mail.ru
}

Doi:10.5901/mjss.2015.v6n4s1p419

\begin{abstract}
The article presents a statistical model of assessment of the symmetry/asymmetry of the generic structure order of the "worldview" in case of the world perception by the Russians, Italians and Frenchmen, based on hyperbolic specific range breakdown. The article shows a higher symmetry of the type structure order of the worldview in case of Italians and Frenchmen as compared to that within the Russian representatives.
\end{abstract}

Keywords: worldview, structure, structure order, specific range breakdown, symmetry, asymmetry.

\section{Introduction}

Integration processes of the contemporary world assume formation of certain "unified" mentality that would not only allow developing the "unified action plan" by the participants of the integrated society, but also give them the chance to act in the context of the "unified vision" of such a plan. From this point of view a special interest is drawn by ethnical mental representations that are considered to be a proof of existence of a certain psychic reality, which is, though initiated by an external input, is formed and supported within the agent and which is generally called as "worldview". Ethno-cultural specifics of the "worldview" has been acknowledged by the global science since quite a long time, as culture is one of the backbone factors in the studies of the human being. G. Gachev, who paid much attention to the ethnical context of the individual "worldview", assumed that the criterion behavior of any groups or communities is the function of a special ethnical "worldview" [Gachev, 1988]. The presented article is not targeted at providing the detailed analysis of the theoretical research in the present sphere, therefore, only those works will be mentioned here which are one way or another connected to the empirical aspect of the ethnical "worldview" research. We would mention two opposite opinions related to the given problem. R. Redfield [Redfield, 1956] considered the ethnical "worldview" to be empirically cognoscible, and A.I. Hollowell [Hollowell, 1955], however, was convinced in the contrary. Some attempts of empirical research of ethnical "worldview" were undertaken by F. Kluckhohn, F. Strodtbeck [Kluckhohn \&Strodtbeck, 1961] in their description of the basic values, and by A. Pavlovskay [Pavlovskay, 2007] in the examination of a national character. Nevertheless, today we do not have numerous scientific works providing definite proof of either existence or nonexistence of ethno-cultural specifics in the "worldview". One of the reasons for this fact is that the evidential basis lacks a mathematical constituent of the research. This article is exactly aimed at finding of mathematically based criteria which would provide the answer for the mentioned question, based on the studies of test results given by the representatives of Italy, France and Russia.

\section{Theoretical Framework}

One of the mathematically based ways to answer the question of existence or non-existence of ethno-cultural specifics of the "worldview", as we see it, can be related to the study of its generic structure. By generic structure we mean the 
diversity of types and the ratio of frequencies of the types within representatives of various ethnic groups. This approach presumes some answers to a number of questions, three of them are as follows. First, the question about generic elements of the "worldview" structure within the representatives of various ethnic groups. Second, the question about the peculiarities of the "worldview" structure order. By the structure order we mean the correlation of "frequency" (of occurrence) of these types for the representatives of various ethnic groups. And, third, coming from the second, the question about the characteristics of that order. Searching for the detailed answer for the first question, which is connected with classification tasks, as we believe, is a matter of little promise. This can be explained by the fact that definition of the "finite border" of the worldview unit is rather problematic because those borders are diffused. The borders that separate one type of the "worldview" from another one are not universal, and depend on the objectives of a specific research or a view of the scholar. Within the context of this authorial position, and due to the fact that in the exploration of the "worldview" types we used a projective pictorial test "Worldview" in the interpretation of E. Romanova, O. Potemkina [Romanova \& Potemkina, 1992], we based our research on the following classification of the "worldview" types: 1) a "planetary worldview" (an image of the Earth, other planets of the Solar system); 2) a "landscape worldview" (presented as an urban or rural landscape, including people, animals, trees, flowers, etc.); 3) "immediate surroundings" (the situation around a person, his/her house as it is, or a situational vision - whatever comes into the mind); 4) a "mediated, or metaphorical worldview" (presents complicated ontological contents as a complex image); 5) an "abstract, schematic worldview" (characterized by a laconic construction presented as an abstract image, a sign, a symbol). Answering the second question we suggested that the order of the "worldview" generic structure can be described with the following stable hyperbolic range distribution ${ }^{W=\frac{A}{B+x^{\beta}}}$ (1.1), where $W$ is a ranking parameter, $A$ is the approximation coefficient (the maximum value of the generic parameter with Range 1, that is in the first point), $r$ is the range number, $\beta$ is the range index characterizing the shape of the distribution curve, $B$ is the range distortion index. At high values of the variable the distribution looks as follows: ${ }^{W=}=\frac{A}{x^{1+\alpha}}$ (Zipf's law) [Kudrin, 1993]. The generic range distribution in our case is the distribution, where, as a parameter, we consider the number (frequencies) of the test audience who possess one of the above mentioned five types of the "worldview", and the range distribution of those frequencies. Three ideas are at the basis of such an assumption. First, there is a hypothesis in knowledge engineering saying that the order of the cognitive structure is relevant not to the order of the physical world structure, but to the order of the social world [Barnes, 1988]. Second, the tendency of social systems to establish and maintain the "optimal order" has been studied since relatively long ago [Vasilkova, 1999]. Third, there is an opinion saying that the order of the social world can be adequately described with asymmetrical distribution, unlike the physical world, which can be adequately described with symmetric distribution (Gaussian distribution) [Haitun, 2005]. The difference between the asymmetrical generic range distribution and symmetric generic range distribution is in presence of considerably large distribution tail areas which is demonstrated in the value of $\beta$-parameter. Thus, within a large volume of data, at $1<\beta<2$ the stable distribution shows absolute dispersion, at $0<\beta<1$ the stable infinitely divisible distribution has neither dispersion nor theoretical mean value. Only at $\beta>2$ Gaussian distribution acts as the absolute distribution [Kudrin, 1993]. In our research, due to a relatively small volume of the test group data, it would be incorrect to use the mentioned interpretation; therefore, in our interpretation we shall assume the following statements. First, the lower the $\beta$ value is, the higher the probability of large distribution tail areas is. We shall consider this assumption as a criterion of potentiality of a higher "generic diversity" of the "worldview". Second, the lower the $\beta$ value is, the lower the symmetry of generic range distribution of the "worldview" structure is. The answer to the third question is in finding $A$ and $\beta$ parameters. To resolve this problem, the approximation of empirical results has been done by log-log scale alignment method. By definition, the closer the aligned line will be to the "ideal line", the more precisely the empirical distribution will meet the power law. In this regard the slope angle of the right line equals the index of power in this function (the coupling coefficient in the linear equation is $\beta$ in Formula 1.1.). The coupling coefficient in the linear equation defines the slope of the right line towards the abscissa axis; therefore we used it as the second (supplementary to the first one) criterion of symmetry - asymmetry of the generic range distribution of the "worldview" for the representatives of various ethnic groups. Considering the assumption that the closer the angle of inclination to the abscissa axis is to the right angle, the higher the probability of symmetric distribution is (for instance, normal, log-normal distribution, Poisson distribution), we can tell that the tendency of the coupling coefficient in the linear equation towards null increases the asymmetry of the order of the generic range distribution of the "worldview". The A parameter in Formula 1.1 is the power index (exponent) of the free term in the linear equation, the B parameter is the offset of the right line upwards on the ordinate axis after alignment in the log-log scale. 


\section{Hypothesis}

The Russians, Italians and Frenchmen differ in their generic structure of the "worldview"; those differences are identified through the generic range distribution and shown in the index of symmetry - asymmetry of that distribution.

\section{Method}

\subsection{Participants}

The total volume of the test group is 428 persons: Russians - 128, Frenchmen - 162, Italians - 138, including males and females at the age of between 20 and 25 years old. The test persons of all three groups belong to various social categories: students, businessmen, social workers, service sector.

\subsection{Procedure}

Projective pictorial test: "Worldview". The choice of the test was based on the fact that projective methods, unlike verbal methods, give the respondents a more flexible canal enabling them to embody the images of their subjective vision of the world. The requisites: sheets of paper $15 \times 20 \mathrm{~cm}$, pencils, pens. The instruction for the participants: "Draw your "worldview", that is the world, its picture as you imagine it". The drawings of the participants were subsequently assessed. For the analysis of the results we have used the interpretation criteria by E. Romanova and O. Potemkina as described above. The expert estimation of the results has been fulfilled by 6 experts who are the lecturers of the University of Florence (Universita degli Studi di Firenze). The estimation of the opinion consensus has been done with the coefficient

of mean-square contingency: $K=\sqrt{\frac{\varphi^{2}}{\sqrt{\left(\kappa_{1}-1\right)\left(\kappa_{2}-1\right)}}}$, where ${ }^{\varphi^{2}=\sum \frac{\sum \frac{n_{w}{ }^{2}}{n_{x}}}{n_{y}}-1}$ is a pair-conjugate index, $\mathrm{k}_{1}$ is the number of values (groups) of the first descriptor (5 views of the world), $\mathrm{k}_{2}$ is the number of values (groups) of the second descriptor (6 experts). The obtained values of $K$ for all the three empirical groups are higher than 0.96 , what proves the adequate consensus within the experts.

\section{Results}

\subsection{Stage 1}

We have calculated the frequencies of 5 types of the "worldview" for Italians, Russians and Frenchmen. The results of the empirical test are presented in Fig. 1.

Figure 1: Frequencies of "Planetary Worldview", "Landscape Worldview", "Immediate Surrounding", "Mediated, or Metaphorical Worldview" and "Abstract, Schematic Worldview" for the Russians (a), Italians (b) and Frenchmen (c)

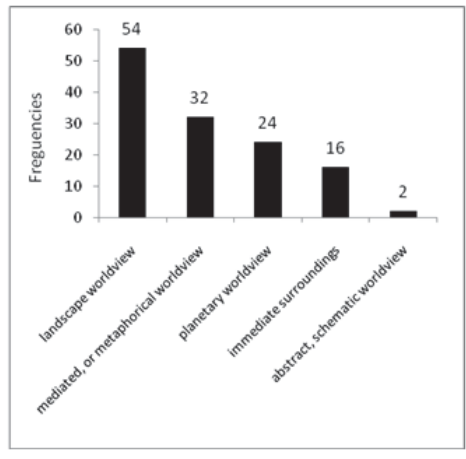

a)

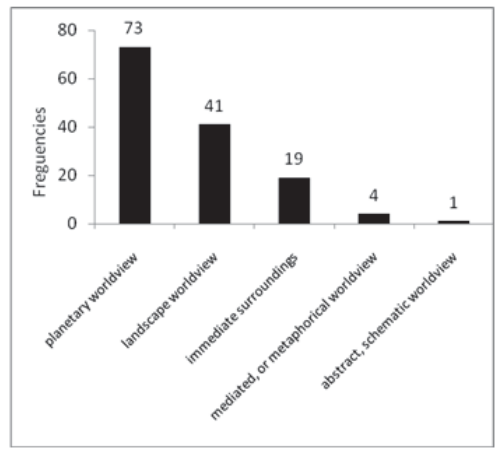

b) 


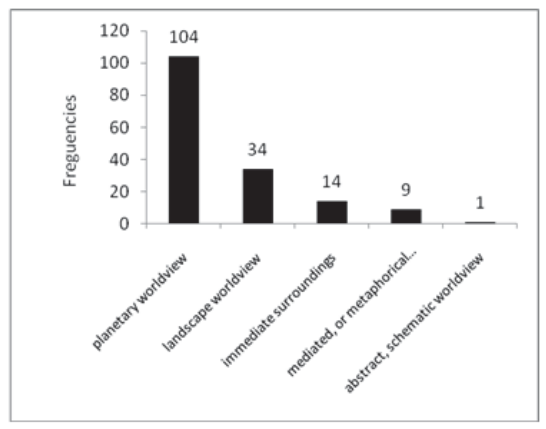

c)

Within the Russian representatives we discovered a more frequent landscape type of the "worldview" - N=54 (42.19\%), and a less frequent abstract type of the "worldview" - $\mathrm{N}=2(1.56 \%)$. The French representatives, unlike the Russians, showed a more frequent planetary type of the "worldview" - $\mathrm{N}=104(64.2 \%)$, and the second frequent type of the "worldview" was landscape - $\mathrm{N}=54$ (21\%), while the abstract type of the "worldview", similar to the Russians, is shown as the most rare case - $\mathrm{N}=1(0.01 \%)$. The Italians, like the Frenchmen, show the planetary type of the "worldview" most frequently - $\mathrm{N}=73(58.2 \%)$, followed by the landscape type of the "worldview", like in the case with the French representatives - $\mathrm{N}=41(29.7 \%)$. The most rare case, similar to the Russians and Frenchmen, is the abstract type of the "worldview" - N=1 (0.01\%).

\subsection{Stage 2}

This stage comprised linier approximation in the log-log scale using SPSS 17.0. The results are plotted in Fig. 2.

Figure 2: Log-log scale linear approximation of the generic range structure of the "worldview" for the Russians (a), Italians (b) and Frenchmen (c) - Results

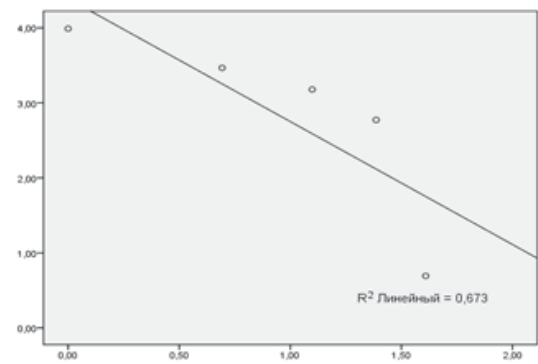

a)

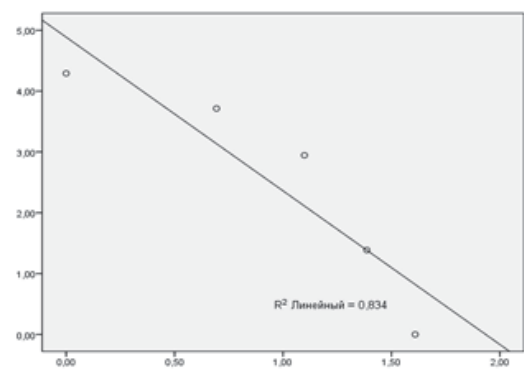

b)

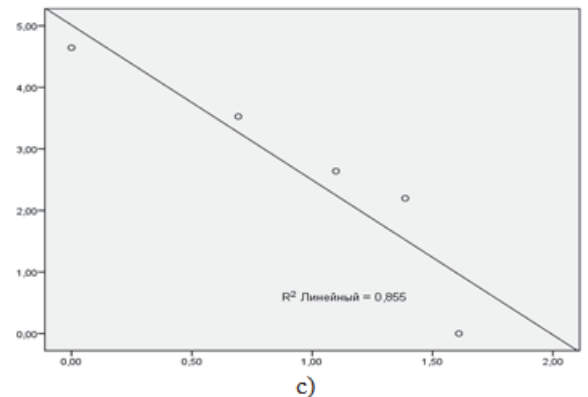

c) 
We have obtained the following linear equations:

for the Italian sampling: $\hat{y}=-2,51 x+4,88\left(R^{2}=.832\right.$, Sig. $\left.\leq .04\right)$;

for the French sampling: $\hat{y}=-2,43 x+4,93\left(R^{2}=.855\right.$, Sig. $\left.\leq .03\right)$;

for the Russian sampling: $\hat{y}=-1,64 x+4,38\left(R^{2}=.637\right.$, Sig. $\left.\leq .08\right)$.

In terms of the RSQ indexes $\left(\mathrm{R}^{2}=.832-.855\right)$ and the allowable error $(\mathrm{Sig} . \leq .05)$ we can conclude that the linier models in the $\log -\log$ scale do the statistically true approximation of the empirical generic range distribution of the "worldview" for the representatives of France and Italy, and have slightly higher allowable error for the representatives of Russia (Sig. s.08).

\subsection{Stage 3}

At this stage we calculated the $A, \beta$ and $B$ parameters. The Italian sampling: ${ }^{W}=\frac{129}{x^{2,51}}$; the French sampling: ${ }^{W}=\frac{136}{x^{2,51}}$; the Russian sampling: $W=\frac{79}{0,69+x^{1,64}}$ (Fig. 2 shows the upward bias of the approximating line for the Russians, which led to the range distortion of $B$ ). The $\beta$-parameter for the French and Italian representatives matched together accurate to two decimate places, which proves that the Italians and Frenchmen have similar orders of the "worldview" structure and the Russians have a different order of the "worldview" structure. Consequently, we calculated theoretical generic range distributions for Italians, Frenchmen and Russians. The results and the empirical and theoretical distributions of the generic range structure of the "worldview" are shown in Fig. 3.

Figure 3: Empirical (a full-line) and theoretical (a dash-line) frequency distribution of the generic range "worldview" structure for Russians (a), Italians (b) and Frenchmen (c).

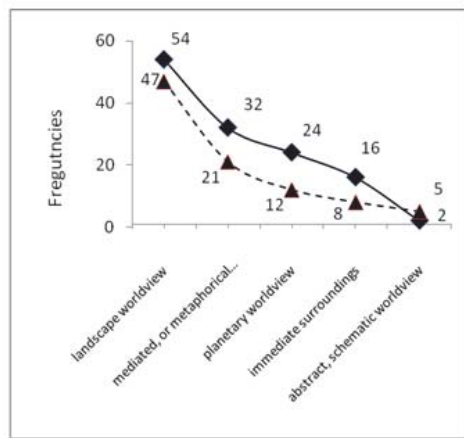

a)

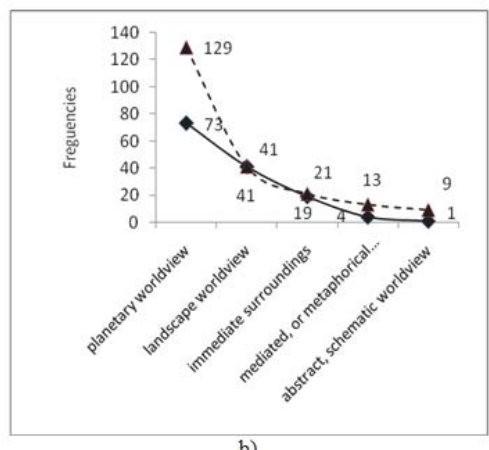

b)

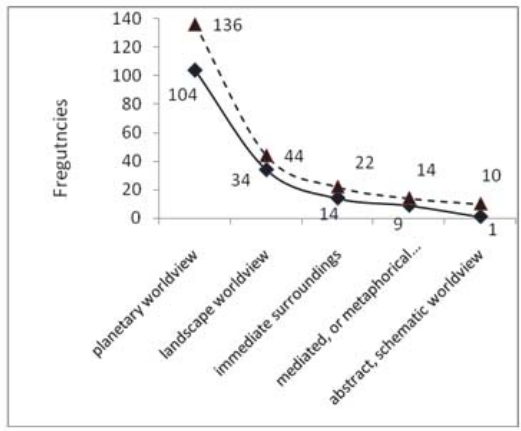

c)

It should be highlighted that in our analysis of the results presented in Fig. 3 we provide only the qualitative analysis without raising the question about the quantitative adequacy of the model. The excess problem (difference between 
frequencies in theoretical and empirical distributions) is corrected quite successfully in parametrical models, but seems problematic, as it is in our case, for non-parametrical models. Deviations from "ideal" hyperbolical distribution are found for all the three samplings. In the case of the Italian and French representatives this is shown in a relatively weak expression of the frequencies of that type which in the generic range distribution of the "worldview" has the first range ("planetary"). In the case with the Russian representatives we see a too strong expression of frequencies of those types which in the generic range distribution of the "worldview" have the second ("mediated or metaphorical") and the third ("planetary") ranges.

\section{Discussion of Results}

The values of frequencies for five types of the "worldview", obtained by the method of a projective pictorial test "Worldview" show that the generic range structures of the "worldview" for the representatives of Russia, France and Italy have both common and specific elements. The common features include occurrence of all those types of the "worldview" ("planetary", "landscape", "immediate surroundings", "mediated or metaphorical", "abstract, schematic" worldviews), as well as the fact that the "abstract, schematic" type is the most rare case within all the test groups. The specific features that can be noted are those that the most frequent case for the generic range "worldview" structure for the Italians and Frenchmen is the "planetary" type of the "worldview", and for the Russians it is the "landscape" type. The point of view of E. Romanova and O. Potemkina is that the dominant "planetary" type of the "worldview" proves predominance of the cognitive world-building, which a) includes classification of the elements of reality; b) implies methods of analysis of reality (explains the causes of events and phenomena, models development of events and phenomena, models the consequences of events). Based on this opinion we can make a conclusion: compared to the Russians, scientism (a higher dependence of the "worldview" on scientific theories) in the "worldview" is more shown within the representatives of France and Italy. A considerably higher value of the angle of inclination to the abscissa axis in the linear model after alignment in log-log scale of the empirical generic range distribution of the "worldview" for the Italians and Frenchmen (with 0.8 difference), compared to the Russians, proves the fact that the structure order of the worldview for the Italians and Frenchmen is closer to the symmetric distribution (Gaussian), such as normal, log-normal distribution, and Poisson distribution. The lower value of the $\beta$-parameter in the generic range distribution of the "worldview" for the Russians can be the criterion of the fact that it possesses the larger asymmetry compared to the Italians and Frenchmen, and that their generic structure has a higher probability of presence of large distribution tail areas. And this is the proof of the fact that the Russians, compared to Frenchmen and Italians, have the "worldview" with potentially higher generic variety.

\section{Conclusions}

1. Frequency distribution of such types of the "worldview" as "planetary", "landscape", "immediate surroundings", "mediated, or metaphorical", and "abstract, schematic" within the Russians, Italians and Frenchmen in its generic structure have shown that there are differences not only in the frequency of the type, but in the range structure as well: the planetary worldview is more common within the Frenchmen and Italians, while the landscape worldview is more typical for the Russians.

2. The criteria which we have applied (angle inclination to the abscissa axis and range index characterizing the shape of the distribution curve) for the estimation of symmetry-asymmetry of the range structure have shown that the generic structure of the "worldview" for the Italians and Frenchmen has a higher symmetry compared to the results of the Russian representatives.

3. A higher probability of the large distribution tail areas in the generic range structure of the "worldview" in case of the Russians proves that their "worldview" has potentially a higher generic variety compared to those of Italian and French.

\section{References}

Barnes B. (1988). The Nature of Power. Cambridge: Policy Press.

Gachev G.D. (1988). National Images of the World. Moscow: Sovetsky Pisatel. 448 p.

Haitun S.D. 2005, The Phenomenon of Man against the Backdrop of Universal Evolution. Moscow, 533 p.

Hallowell A.I. (1955) Culture and Experience. Philadelphia University of Pennsylvania Press.

Kluckhohn F, Strodtbeck F. (1961) Variations in Value Orientations. New York: Row, Peterson and Co., USA. 187 p.

Kudrin B.I. (1993). Introduction to Technetics. 2nd edition, revised, updated. Tomsk: Tomsk University Edition. 552 p. 
Redfield R. (1955). The Little Community. Viewpoints for the Study of a Human Whole. Uppsala and Stockholm: Almovist and Wiksells. $394 \mathrm{p}$.

Romanova E.S., Potemkina O.F. Graphic Methods in Psychological Diagnostics. Moscow: Didakt, 1992. - 256 p.

Vasilkova V.V. (1999). Order and Chaos in Development of Social Systems. (Synergetics and Theory of Social Self-organization). St. Petersburg: Lan Publishing, 1999. 480 p.

Pavlovskay A. (2007) Peculiarities of National Characters of Italians, Englishmen, Germans, Norwegians and Finns, Americans, Icelanders. Moscow: Moscow State University, Faculty of Foreign Languages. - 172 p. 